\title{
A Deep Learning Approach for Motor Imagery EEG Signal Classification
}

\author{
Shiu Kumar ${ }^{1,2}$, Alok Sharma ${ }^{2,3,4,5, *}$, Kabir Mamun ${ }^{2}$, Tatsuhiko Tsunoda ${ }^{4,5,6}$ \\ ${ }^{1}$ Department of Electronics Engineering, Fiji National University, Suva, Fiji \\ ${ }^{2}$ School of Engineering \& Physics, University of the South Pacific, Suva, Fiji \\ ${ }^{3}$ Institute for Integrated and Intelligent Systems (IIIS), Griffith University, Brisbane, Australia \\ ${ }^{4}$ RIKEN Center for Integrative Medical Sciences, Yokohama 230-0045, Japan \\ ${ }^{5}$ CREST, JST, Yokohama 230-0045, Japan \\ ${ }^{6}$ Medical Research Institute, Tokyo Medical and Dental University, Tokyo 113-8510, Japan \\ shiu.kumar@fnu.ac.fj; alok.fj@gmail.com
}

\begin{abstract}
Over the last few decades, the use of electroencephalography (EEG) signals for motor imagery based brain-computer interface (MI-BCI) has gained widespread attention. Deep learning have also gained widespread attention and used in various application such as natural language processing, computer vision and speech processing. However, deep learning has been rarely used for MI EEG signal classification. In this paper, we present a deep learning approach for classification of MI-BCI that uses adaptive method to determine the threshold. The widely used common spatial pattern (CSP) method is used to extract the variance based CSP features, which is then fed to the deep neural network for classification. Use of deep neural network (DNN) has been extensively explored for MI-BCI classification and the best framework obtained is presented. The effectiveness of the proposed framework has been evaluated using dataset IVa of the BCI Competition III. It is found that the proposed framework outperforms all other competing methods in terms of reducing the maximum error. The framework can be used for developing BCI systems using wearable devices as it is computationally less expensive and more reliable compared to the best competing methods.
\end{abstract}

Keywords-brain-computer interface (BCI); common spatial pattern (CSP); deep neural network (DNN); electroencaphalography (EEG), motor imagery (MI).

\section{INTRODUCTION}

In recent years, the use of brain signals have been extensively explored for various applications with major focus to the field of biomedical engineering [1-4]. A BCI system, also referred to as brain-machine interaction, bridges the gap between humans and computers by translating thoughts into commands, which can be used to communicate with the external devices [5-8] or used for disease diagnosis $[3,9,10]$. A BCI system could be beneficial in restoring valuable functions of severely disable people. In BCI systems based on EEG techniques, usually noninvasive sensors are placed on the scalp of the user for detecting the changes in the electrical potentials that are originated by the neurons. Due to low cost and portability, EEG is traditionally used in comparison with other noninvasive methods such as magneto-encephalography (MEG), position emission tomography (PET), and functional magnetic resonance imaging (fMRI) systems. Use of EEG is also preferred over invasive method such as electrocorticography $(\mathrm{ECoG})$ as the later requires a brain surgery for setting up the montage.

The major challenge in the classification of MI EEG signals arises due to the fact that the brain signals that are recorded are very small in amplitude. Therefore, events such as eye blink, eye movement, muscular movements, teeth grinding and heart rhythm interfere with the EEG signal resulting in a signal having low signal to noise ratio (SNR). This prevents the decoding system to correctly decode the user thoughts. Various techniques have been proposed by the scientific community aiming to improve the temporal filtering methods $[11,12]$, spatial filtering [13-15], feature extraction $[16,17]$ and feature selection [18-21] techniques, dimensionality reduction techniques [22-24] and classification algorithms [25-28]. Several feature extraction techniques such as power spectral density (PSD), common spatial pattern (CSP) [29, 30], statistical features, selforganizing maps (SOM), correlation, spectral coherence [31] and information entropy [17, 32] have been studied. Classifiers such as support vector machine (SVM) [26, 33], k-nearest neighbors (KNN) [34-36], random forest (RF) [37], etc. have been explored for classification of MI-EEG signals.

Although a vast range of studies on a number of different aspects of the MI based BCI's have been carried out, the detection and modeling of brain signals using artificial intelligence and machine learning techniques remains a big challenge and many open research questions still exists. Deep learning techniques have also increasingly gained attention in the field of machine learning and artificial intelligence. It has been widely explored and used as solutions for engineering problems such as speech recognition [38, 39], computer vision [40, 41] and natural language processing [42]. However, the use of deep learning techniques has not been widely explored for MI based BCI systems. Only a number of studies has been carried out to 
explore the use of deep learning techniques for EEG signal classification [43-46].

In this paper, we explore the use of deep learning techniques for MI-EEG signal classification. Several frameworks have been evaluated and the framework that performed well in comparison with other competing methods is presented in detail. The rest of the paper is organized as follows. Section II briefly discusses some of the research work that has been carried out in the related field. The description of the dataset used and the proposed framework is described in Section III. In section IV, the proposed framework is evaluated and compared with other competing methods while section $\mathrm{V}$ highlights the important findings of this paper and makes some future insights.

\section{RELATED WORKS}

A BCI system could be beneficial in restoring valuable functions of severely disabled people, used for detection/diagnosis of disease such as seizure detection and used for entertainment. Filter band selection, optimizing spatial filters, feature extraction and classification algorithms have been widely explored to develop improved BCI systems. BCI systems such as wheelchair controllers $[6,47]$, word speller programs [48] and automatic emotion recognition systems $[49,50]$ have been successfully studied and developed. A BCI system bridges the gap between human and machines interactions by controlling external devices via thought without any muscle activity.

The conventional CSP method employs a single fixed filter bank with CSP variance based features and obtained promising results. CSP has been widely used for EEG signal classification. A common spatio-spectral pattern (CSSP) [51] framework has been proposed that enhances the performance of the conventional CSP. In CSSP, CSP is applied to the signals combined with its time delayed signals to obtain the finite impulse response filter (FIR) coefficients. Different spectral patterns are computed for each channel in the CSSP framework.

In [30], a sub-band common spatial pattern (SBCSP) method is presented (refer Fig. 1) in which the authors have expanded the method of conventional CSP. The raw multichannel EEG signal is decomposed into multiple frequency bands called sub-bands. The CSP variance based features are then extracted for each of the sub-bands and linear discriminant analysis (LDA) is performed on each of the feature sets of the different sub-bands in order to reduce the dimensionality of the feature sets. They have applied the method to two class problem, therefore one dimension feature set is obtained for each set of sub-band on which LDA is performed. Finally, the set of features are fused together and a SVM is used for classification. This SBCSP method performed better than the conventional CSP approach.

As the number of features increases, the possibility to have redundant features also increases and sometimes the overall performance reduces. This is the case in SBCSP and to tackle this problem, a filter bank CSP (FBCSP) [29] method has been proposed. The FBCSP method is similar to
SBCSP, however, after performing CSP on the sub-bands, instead of performing LDA, the CSP features of the subbands are combined and feature selection have been performed. The authors in this method evaluated various feature selection methods and classifiers. The FBCSP method performed better than the conventional CSP and SBCSP.

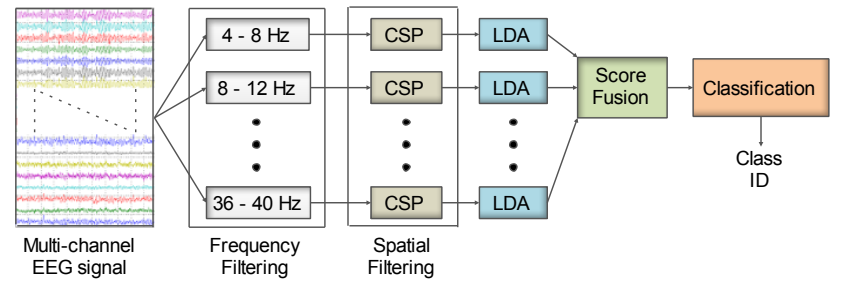

Fig. 1. The framework for SBCSP [30] method.

The use of nine sub-bands increases the computational burden of the SBCSP and FBCSP methods. To overcome this issue, a discriminative filter band CSP (DFBCSP) has been proposed in [11]. In DFBCSP, Fisher's ratio of the spectral power of channel $\mathrm{C} 3$ or $\mathrm{C} 4$ is used to determine the four most discriminating frequency bands from a set of 12 frequency bands. CSP is then performed on each of the selected 4 sub-bands separately and the variance based CSP features are extracted. The features from all bands are combined and fed to a SVM classifier. The DFBCSP method thus reduces the computational burden of the SBCSP and FBCSP frameworks as only 4 sub-bands are used compared to that of 9 used by SBCSP and FBCSP. It also outperforms all the other methods achieving the highest classification accuracy.

A few researchers have also focused on employing deep learning for EEG signal classification. In [44], a deep belief network (DBN) is employed for classification of emotions using EEG signals. Differential entropy features are extracted from multichannel EEG signals and used for training the deep belief network. A hidden markov model (HMM) is also integrated to the output of the DBN in order to capture a reliable emotional stage switching with higher accuracy. The DBN method performed better in comparison with the state-of-the-art methods. A deep learning method for classification of EEG data based on motor imagery have been presented in [52]. The authors employed the method for EEG based left and right hand motor imagery tasks. They firstly trained a weak classifier by DBN using certain single channel data and then combined the trained weak classifiers using the AdaBoost algorithm. A number of restricted Boltzmann machines (RBM) were stacked on top of each other to form the DBN structure. Contrastive divergence (CD) algorithm was used for training the DBN network. The authors reported that better performance was obtained using 8 hidden layers with improvement of $4-6 \%$ in certain cases in comparison with SVM.

In [53], a new deep learning scheme based on RBM is presented. Wavelet packet decomposition (WPD) and fast Fourier transform (FFT) were employed for obtaining the frequency domain representation of the EEG signals. These were used to train $3 \mathrm{RBMs}$ and then a softmax regression 
output layer was stacked forming a four-layer network. This is known as frequential deep belief network (FDBN). The FDBN was fine tuned using conjugate gradient and backpropagation methods. The FDBN was evaluated using public benchmark datasets and significant performance improvement have been shown compared to the state-of-theart methods.

In this paper, we propose a novel deep learning method that accepts CSP features as inputs. Only a single filter band is employed for filtering the raw EEG signal in order to keep the computational complexity of the system to a minimum.

\section{Methodology}

\section{A. EEG Data Description}

The public benchmark Dataset IVa from BCI competition III provided by Fraunhofer FIRST (intelligent data analysis group) have been used $[54,55]$ to evaluate the performance of the proposed CSP based DNN (CSP-DNN) framework and referred to as dataset from here onwards. The dataset consists of motor imagery EEG signals for right hand and left foot recorded from five subjects using 118 channels. The five subjects are referred to as $a a, a l, a v, a w$ and $a y$. The down sampled signals of $100 \mathrm{~Hz}$ have been used. The dataset contains 280 trials (140 for each class) for each subject. More details of the dataset can be obtained at the web-link: http://www.bbci.de/competition/iii/.

\section{B. The proposed CSP based DNN framework}

The block diagram of the proposed CSP-DNN framework is shown in Fig. 2. The raw EEG data is first filtered by bandpass filter. Spatial filtering using CSP is then performed on the bandpass filtered data and the CSP variance based features are extracted. The DNN is then trained using the extracted features and the trained DNN is used to classify the test data. The following sections explain the different stages of the proposed CSP-DNN framework in detail.

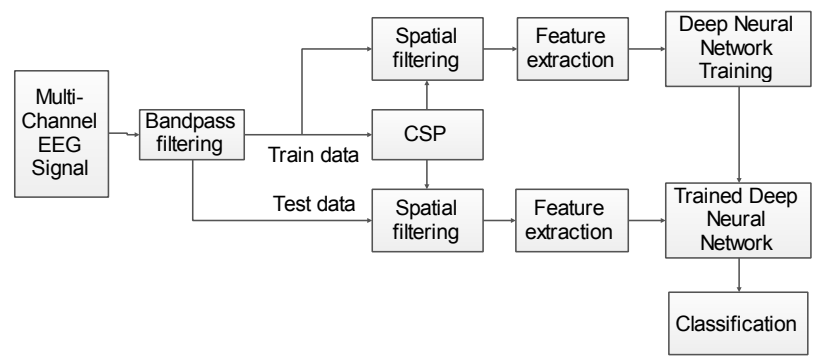

Fig. 2. Framework of the proposed CSP-DNN framework.

\section{CSP feature extraction}

CSP have been widely used in MI-BCI systems for projecting the data to a new time series that has maximum discrimination between the different MI tasks. Consider the two class problem having EEG samples $X_{n, i} \in R^{C \times T}$, where $n$ denotes the $n$-th trial of $i$-th class $(i \in\{1,2\}), \mathrm{C}$ is the number of channels and $\mathrm{T}$ is the number of sample points. Each trial data is spatially filtered using (1), $W_{C S P}$ is the spatial filter, and $Z_{n, i}$ is the spatially projected data. The CSP variance based features are then extracted from each trial using (2), where $f_{i}$ is the $i$-th feature, and $\operatorname{var}\left(Z_{m}\right)$ denotes the variance of the $m$-th row of $Z$. The features $f_{i}$ are then combined to form the feature vector of each trial. Each trial feature vector of the train data is stacked together to form the train feature matrix. The test feature matrix is obtained in the same way. These feature matrices are used for training and testing of the DNN. A detailed explanation of CSP algorithm can be found in [56].

$$
Z_{n, i}=W_{C S P}^{T} X_{n, i}
$$

$$
f_{i}=\log \left(\frac{\operatorname{var}\left(Z_{i}\right)}{\sum_{n=1}^{2 m} \operatorname{var}\left(Z_{n}\right)}\right)
$$

\section{The proposed DNN structure}

Deep learning techniques are widely used in speech, image, signal, video, and text mining and recognition, and are breaking the records by improving state of the art accuracies. Recently remarkable improvements have been made by deep learning techniques in the tasks of classification and representation learning. In this work, we employed a 4-layer DNN comprising of the input layer, 2 hidden layers, and an output layer as shown in Fig. 3.

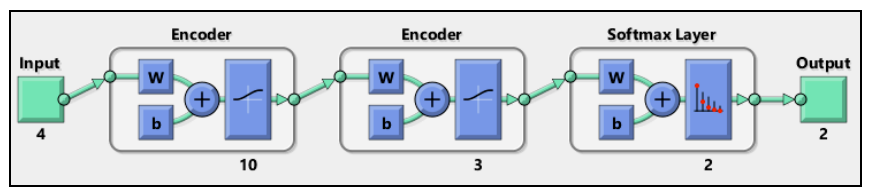

Fig. 3. The DNN structure that has been adopted (source: MATLAB)

The 4 dimensional CSP feature vector forms the input to the DNN. The two hidden layers are the encoders having 10 and 3 nodes respectively while the final layer is the softmax layer with 2 nodes (this structure was selected from many other structures as discussed in Section IV). The auto encoders are used to learn features in an unsupervised way. The input of the first encoder is the 4 dimensional feature vectors and the output is the same. Thus, the 10 hidden nodes after training produces 10 new learned features. An auto encoder comprises of an encoder and a decoder. The encoder learns the features while the decoder maps the learned features back to the input. These learned features are fed to the second encoder which learns 3 new features (same as the number of nodes) in a similar manner. No scaling of data has been done and logistic sigmoid function and pure line transfer function have been used for the encoder and decoder, respectively. The mean squared error is used as the loss function to evaluate the performance during the training process. The softmax layer is used for classification and scaled conjugate gradient descent algorithm has been used for training the softmax layer. Cross-entropy has been used as the loss function. The softmax layer uses the actual target class during training and thus is a supervised technique. All algorithms were implemented using MATLAB. 


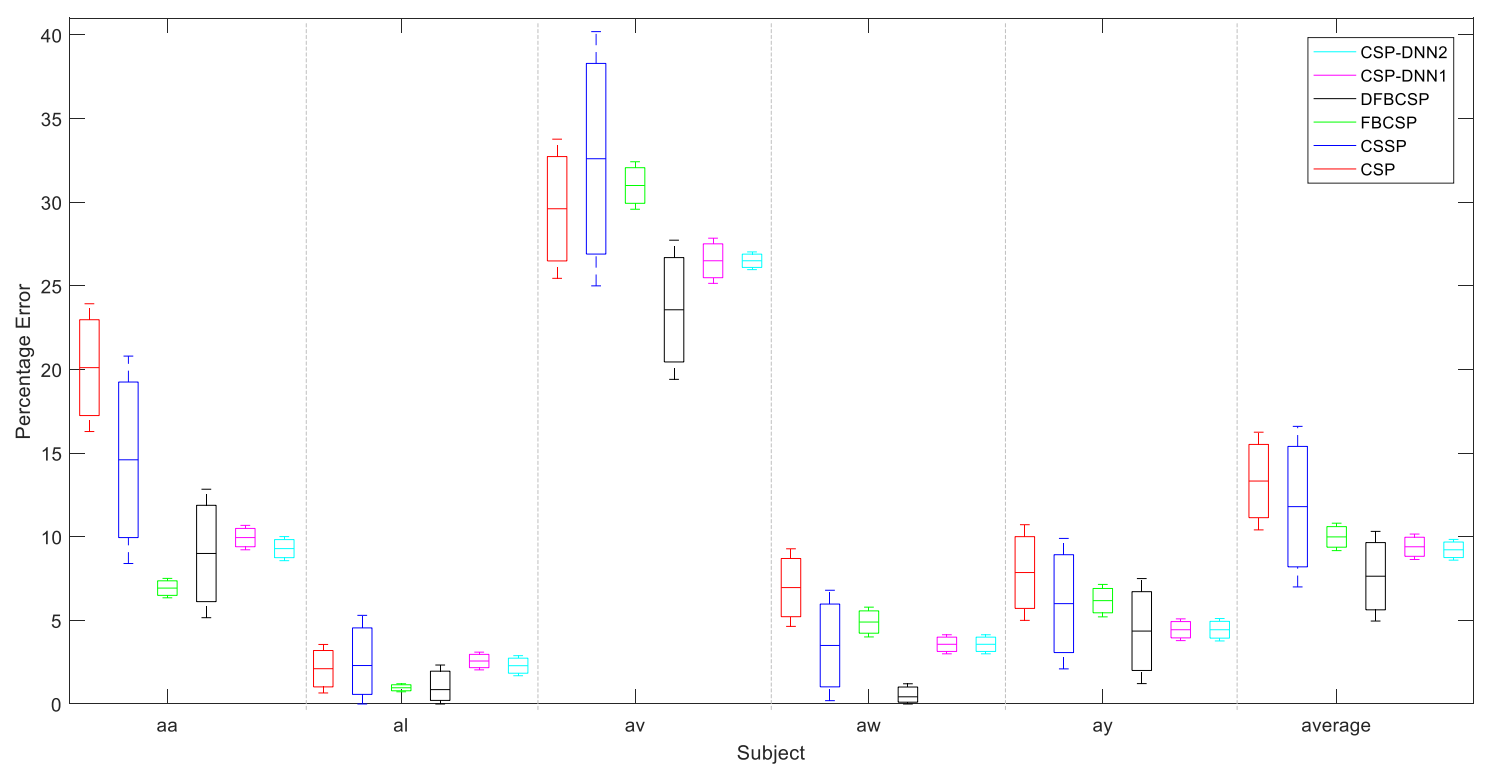

Fig. 4. Box plot showing the average 10x10-fold cross validation errors for different methods using public benchmark dataset IVa of BCI Competition III.

\section{RESUlts AND Discussions}

A number of experiments have been carried out to obtain the CSP-DNN framework that gives promising results. Four different frameworks were evaluated in this work. In the first framework, the bandpass filtered raw EEG data was directly used to train the DNN with two hidden layers. In the second framework, the bandpass filtered raw data was spatially filtered using CSP spatial filter and the spatially projected data was used as the input to the DNN. In the third framework, the bandpass filtered raw EEG data was directly used to learn a lower dimension data in an unsupervised way using auto encoders. The lower dimensional data were then spatially filtered using the CSP spatial filter and the spatially filtered data was then used to learn another DNN. All these frameworks did not perform well compared to the state-of-the-art methods and were not adopted. The fourth framework is the proposed CSP-DNN that has been presented in Section III.

Experiments were conducted using several different node configurations and the configuration that gave promising results has been adopted. In the conventional CSP method, a single wide bandpass filter is used. While this keeps the computational complexity of the system low and produced promising results, other state of the art methods such as FBCSP and DFBCSP performed better at the expense of increased computational complexity employing multiple filter banks in conjunction with either feature selection or band selection algorithms. The aim of this research work was to develop a BCI system that can compete with the state-of-the-art methods and is computationally less expensive. Therefore, we have employed a single filter bank. However, instead of using a fixed bandpass filter as used in conventional CSP, we tuned the filters using 10 -fold cross validation method.

In the CSP algorithm, the spatial filter $W_{C S P}$ is made by selecting the first and last $m$ columns of the CSP projection matrix. In this work $m=2$ have been used. Other values of $m$ were also evaluated, however, using $m=2$ produced the desirable results. Thus, for each trial a $4(2 \mathrm{~m})$ dimensional feature vector is obtained. The features of the training set were used to train the DNN and the features from the test set were then classified using the trained weights of DNN. The DNN structure has been adopted after a series of experiments in which different number of nodes in each of the hidden layers (encoders) were tested. Using 10 nodes for the first encoder and 3 nodes for the second encoder gave optimal results and thus have been adopted. The effectiveness of the proposed CSP-DNN framework has been evaluated using $10 \times 10$ fold cross validation method and the results obtained are shown in Fig. 4. The CSP-DNN framework has been tested with two different settings. In the first setting (CSP-DNN1), a threshold of 0.50 has been used for classification whereas for the second setting (CSPDNN2) an adaptive method was used for selecting the threshold parameter. Ten-fold cross validation was performed on the training data to select the best threshold value and later used to classify the test data. It has been noted that using the adaptive method of selecting the threshold resulted in an improvement in both the average mean error and maximum error.

Overall, the proposed CSP-DNN framework performed well and promising results have been obtained. The proposed CSP-DNN method has successfully reduced the overall maximum error producing a more reliable result. Although the DFBCSP method achieved the lowest average error, the maximum error is quite high. It can be noted that the proposed method also reduced the maximum error for subjects $a y$ and $a v$ in comparison with other methods.

In terms of computational complexity, the proposed method uses a single filter bank in comparison with FBCSP and DFBCSP methods that employ multiple filter banks. Thus, employing a single filter bank has reduced the computational complexity of the proposed CSP-DNN 
framework in comparison with the competing methods, FBCSP and DFBCSP. Comparing CSP, CSSP and CSPDNN framework all methods use a single filter bank, however, the CSP-DNN method outperforms both the methods. The proposed CSP-DNN framework has outperformed all methods except DFBCSP in terms of average error. However, it should also be taken into account that DFBCSP employs band selection and later uses 4 selected sub-bands for further processing. Therefore, DFBCSP has obtained a lower average error at the expense of increased computation cost. On the other hand, the CSPDNN method is computationally less expensive and competes well with the best performing method, DFBCSP.

\section{CONCLUSION}

A number of experiments have been conducted on several different frameworks with different parameter settings and a CSP-DNN framework has been proposed, which outperformed the state-of-the-art methods in terms of reducing maximum error. The proposed system is more reliable as it achieved the lowest maximum error and is computationally efficient. Thus the CS-DNN framework is well suited to applications such as wearable devices that require algorithms that are computationally less expensive and can last longer when powered using batteries.

In this work, we aimed to keep the complexity of the system to a minimum and thus employed a single filter bank. However, using the CSP-DNN in parallel on multiple filter banks and incorporating a majority voting system may further enhance the performance of the system, which is left as future work. Also, in this work we limited the number of hidden layers to 2 . Therefore, in future we will carry out more research and investigate the impact of increasing the number of hidden layers in a quest to obtain an improved system further.

\section{AKNOWLEDGMENT}

The authors would like to thank the College Research Committee (CRC) of Fiji National University, Faculty Research Committee (FRC) of the University of the South Pacific and RIKEN Center for Integrative Medical Sciences for supporting and funding this research work.

\section{REFERENCES}

[1] T. Yu, J. Xiao, F. Wang, R. Zhang, Z. Gu, A. Cichocki, et al., "Enhanced Motor Imagery Training Using a Hybrid BCI With Feedback," IEEE Transactions on Biomedical Engineering, vol. 62, pp. 1706-1717, 2015.

[2] R. Salazar-Varas, A. Costa, A. Úbeda, E. Iáñez, and J. M. Azorín, "Changes in brain activity due to the sudden apparition of an obstacle during gait," in 7th International IEEE EMBS Neural Engineering Conference (NER), ed Montpellier, France: IEEE, 2015.

[3] M. Z. Parvez, M. Paul, and M. Antolovich, "Detection of Prestage of Epileptic Seizure by Exploiting Temporal Correlation of EMD Decomposed EEG Signals," Journal of Medical and Bioengineering, vol. 4, pp. 110-116, 2015.

[4] Y. Liu, M. Li, H. Zhang, H. Wang, J. Li, J. Jia, et al., "A tensor-based scheme for stroke patients' motor imagery EEG analysis in BCI-FES rehabilitation training," Journal of Neuroscience Methods, vol. 222, pp. 238-249, 1/30/ 2014.
[5] S. Ramesh, M. G. Krishna, and M. Nakirekanti, "Brain Computer Interface System for Mind Controlled Robot using Bluetooth," International Journal of Computer Applications, vol. 104, pp. 20-23, October 2014.

[6] R. S. Naveen and A. Julian, "Brain computing interface for wheel chair control," in Fourth International Conference on Computing, Communications and Networking Technologies (ICCCNT), 2013, pp. $1-5$.

[7] A. C. Lopes, G. Pires, and U. Nunes, "Assisted navigation for a brainactuated intelligent wheelchair," Robotics and Autonomous Systems, vol. 61, pp. 245-258, 2013.

[8] B. Choi and S. Jo, "A Low-Cost EEG System-Based Hybrid BrainComputer Interface for Humanoid Robot Navigation and Recognition," PLOS one, vol. 8, p. e74583, 2013.

[9] A. Sengur, Y. Guo, and Y. Akbulut, "Time-frequency texture descriptors of EEG signals for efficient detection of epileptic seizure," Brain Informatics, vol. 3, pp. 101-108, 2016.

[10]H. R. A. Ghayab, Y. Li, S. Abdulla, M. Diykh, and X. Wan, "Classification of epileptic EEG signals based on simple random sampling and sequential feature selection," Brain Informatics, vol. 3, pp. 85-91, 2016.

[11] K. P. Thomas, G. Cuntai, C. T. Lau, A. P. Vinod, and A. Kai Keng, "A New Discriminative Common Spatial Pattern Method for Motor Imagery Brain Computer Interfaces," IEEE Transactions on Biomedical Engineering, vol. 56, pp. 2730-2733, 2009.

[12] Q. Wei and Z. Wei, "Binary particle swarm optimization for frequency band selection in motor imagery based brain-computer interfaces," Bio-Medical Materials and Engineering, vol. 26, pp. S1523-S1532, 2015.

[13] A. S. Aghaei, M. S. Mahanta, and K. N. Plataniotis, "Separable Common Spatio-Spectral Patterns for Motor Imagery BCI Systems," IEEE Transactions on Biomedical Engineering, vol. 63, pp. 15-29, 2016.

[14] X. Li, X. Lu, and H. Wang, "Robust common spatial patterns with sparsity," Biomedical Signal Processing and Control, vol. 26, pp. 52$57,4 / / 2016$

[15] W. Wu, Z. Chen, X. Gao, Y. Li, E. N. Brown, and S. Gao, "Probabilistic Common Spatial Patterns for Multichannel EEG Analysis," Pattern Analysis and Machine Intelligence, IEEE Transactions on, vol. 37, pp. 639-653, 2015.

[16] A. Zaitcev, G. Cook, L. Wei, M. Paley, and E. Milne, "Feature extraction for BCIs based on electromagnetic source localization and multiclass Filter Bank Common Spatial Patterns," in Engineering in Medicine and Biology Society (EMBC), 2015 37th Annual International Conference of the IEEE, 2015, pp. 1773-1776.

[17] A. Zhang, B. Yang, and L. Huang, "Feature Extraction of EEG Signals Using Power Spectral Entropy," in International Conference on BioMedical Engineering and Informatics, Sanya, 2008, pp. 435 - 439.

[18] A. Sharma, S. Imoto, and S. Miyano, "A Top-r Feature Selection Algorithm for Microarray Gene Expression Data," IEEE/ACM Trans. Comput. Biol. Bioinformatics, vol. 9, pp. 754-764, 2012.

[19] J. Luo, Z. Feng, J. Zhang, and N. Lu, "Dynamic frequency feature selection based approach for classification of motor imageries," Computers in Biology and Medicine, vol. 75, pp. 45-53, 8/1/ 2016.

[20] A. Sharma, K. K. Paliwal, S. Imoto, and S. Miyano, "A feature selection method using improved regularized linear discriminant analysis," Machine Vision and Applications, vol. 23, pp. 775-786, April 2014.

[21] A. Sharma, K. K. Paliwal, S. Imoto, and S. Miyano, "A feature selection method using improved regularized linear discriminant analysis," Machine Vision and Applications, vol. 25, pp. 775-786, 2014.

[22] A. Sharma and K. K. Paliwal, "A deterministic approach to regularized linear discriminant analysis," Neurocomputing, vol. 151, Part 1, pp. 207-214, 3/3/ 2015.

[23]A. Sharma and K. K. Paliwal, "Rotational Linear Discriminant Analysis Technique for Dimensionality Reduction," IEEE Transactions on Knowledge and Data Engineering, vol. 20, pp. 1336$1347,2008$. 
[24]A. Sharma and K. K. Paliwal, "A Gradient Linear Discriminant Analysis for Small Sample Sized Problem," Neural Processing Letters, vol. 27, pp. 17-24, 2008.

[25]H. Raza, H. Cecotti, Y. Li, and G. Prasad, "Adaptive learning with covariate shift-detection for motor imagery-based brain-computer interface," Soft Computing, vol. 20, pp. 3085-3096, 2016.

[26] X. Li, X. Chen, Y. Yan, W. Wei, and Z. J. Wang, "Classification of EEG Signals Using a Multiple Kernel Learning Support Vector Machine," Sensors, vol. 14, pp. 12784-12802, July 2014.

[27] A. Adam, M. I. Shapiai, M. Z. Mohd Tumari, M. S. Mohamad, and M. Mubin, "Feature Selection and Classifier Parameters Estimation for EEG Signals Peak Detection Using Particle Swarm Optimization," The Scientific World Journal, vol. 2014, p. 13, 2014.

[28] A. Sharma, K. K. Paliwal, and G. C. Onwubolu, "Class-dependent PCA, MDC and LDA: A combined classifier for pattern classification," Pattern Recognition, vol. 39, pp. 1215-1229, 7// 2006.

[29] K. K. Ang, Z. Y. Chin, H. Zhang, and C. Guan, "Filter Bank Common Spatial Pattern (FBCSP) in Brain-Computer Interface," in IEEE International Joint Conference on Neural Networks (IEEE World Congress on Computational Intelligence), Hong Kong, 2008, pp. 2390 $-2397$.

[30] Q. Novi, G. Cuntai, T. H. Dat, and X. Ping, "Sub-band Common Spatial Pattern (SBCSP) for Brain-Computer Interface," in 3rd International IEEE/EMBS Conference on Neural Engineering, 2007, pp. 204-207.

[31] D. L. Rocca, P. Campisi, B. Vegso, P. Cserti, G. Kozmann, F. Babiloni, et al., "Human Brain Distinctiveness Based on EEG Spectral Coherence Connectivity," IEEE Transactions on Biomedical Engineering, vol. 61, pp. 2406-2412, September 2014.

[32] M. Sabeti, S. Katebi, and R. Boostani, "Entropy and complexity measures for EEG signal classification of schizophrenic and control participants," Artificial Intelligence in Medicine, vol. 47, pp. 263-274, November 2009

[33] N.-H. Liu, C.-Y. Chiang, and H.-C. Chu, "Recognizing the Degree of Human Attention Using EEG Signals from Mobile Sensors," Sensors, vol. 13, pp. 10273-10286, August 2013.

[34]L. Brown, B. Grundlehner, and J. Penders, "Towards wireless emotional valence detection from EEG," in Annual International Conference of the IEEE Engineering in Medicine and Biology Society (EMBC), 2011, pp. 2188-2191.

[35]X. Haiyan and K. N. Plataniotis, "Affect recognition using EEG signal," in IEEE 14th International Workshop on Multimedia Signal Processing (MMSP), 2012, pp. 299-304.

[36] E. Parvinnia, M. Sabeti, M. Z. Jahromi, and R. Boostani, "Classification of EEG Signals using adaptive weighted distance nearest neighbor algorithm," Journal of King Saud University Computer and Information Sciences, vol. 26, pp. 1-6, 2014.

[37]F. Akram, H.-S. Han, and T.-S. Kim, "A P300-Based Word Typing Brain Computer Interface System Using a Smart Dictionary and Random Forest Classifier," in The Eighth International MultiConference on Computing in the Global Information Technology, 2013, pp. 106-109.

[38] M. Ahmed, P. C. Shill, K. Islam, M. A. S. Mollah, and M. A. H. Akhand, "Acoustic modeling using deep belief network for Bangla speech recognition," in 2015 18th International Conference on Computer and Information Technology (ICCIT), 2015, pp. 306-311.

[39] G. Hinton, L. Deng, D. Yu, G. E. Dahl, A. r. Mohamed, N. Jaitly, et al., "Deep Neural Networks for Acoustic Modeling in Speech Recognition: The Shared Views of Four Research Groups," IEEE Signal Processing Magazine, vol. 29, pp. 82-97, 2012.

[40] A. P. James, D. Al-Jumeily, S. M. Thampi, T. Dobhal, V. Shitole, G. Thomas, et al., "Human Activity Recognition using Binary Motion Image and Deep Learning," Procedia Computer Science, vol. 58, pp. 178-185, January 2015.

[41]A. Knittel and A. D. Blair, "An Abstract Deep Network for Image Classification," in AI 2012: Advances in Artificial Intelligence: 25th Australasian Joint Conference, Sydney, Australia, December 4-7, 2012. Proceedings, M. Thielscher and D. Zhang, Eds., ed Berlin, Heidelberg: Springer Berlin Heidelberg, 2012, pp. 156-169.
[42] T. s. Mikolov, A. Deoras, S. Kombrink, L. s. Burget, and J. H. Cernock, "Empirical evaluation and combination of advanced language modeling techniques," presented at the INTERSPEECH ISCA, 2011.

[43] S. Stober, D. J. Cameron, and J. A. Grahn, "Using convolutional neural networks to recognize rhythm stimuli from electroencephalography recordings," in Advances in Neural Information Processing Systems (NIPS), 2014, pp. 1449-1457.

[44] W. L. Zheng, J. Y. Zhu, Y. Peng, and B. L. Lu, "EEG-based emotion classification using deep belief networks," in 2014 IEEE International Conference on Multimedia and Expo (ICME), 2014, pp. 1-6.

[45] S. M. Plis, D. R. Hjelm, R. Salakhutdinov, E. A. Allen, H. J. Bockholt, J. D. Long, et al., "Deep learning for neuroimaging: a validation study," Frontiers in Neuroscience, vol. 8, 2014-August-20 2014.

[46] S. Jirayucharoensak, S. Pan-Ngum, and P. Israsena, "EEG-Based Emotion Recognition Using Deep Learning Network with Principal Component Based Covariate Shift Adaptation," The Scientific World Journal, vol. 2014, p. 10, 2014.

[47] L. Cao, J. Li, H. Ji, and C. Jiang, "A hybrid brain computer interface system based on the neurophysiological protocol and brain-actuated switch for wheelchair control," Journal of Neuroscience Methods, vol. 229, pp. 33-43, 5/30/ 2014.

[48]F. Akram, M. K. Metwally, H. Hee-Sok, J. Hyun-Jae, and K. TaeSeong, "A novel P300-based BCI system for words typing," in International Winter Workshop on Brain-Computer Interface (BCI), 2013, pp. 24-25.

[49] N. Jatupaiboon, S. Pan-ngum, and P. Israsena, "Real-Time EEG-Based Happiness Detection System," The Scientific World Journal, vol. 2013 p. 618649, July 2013.

[50] U. Wijeratne and U. Perera, "Intelligent emotion recognition system using electroencephalography and active shape models," in IEEE EMBS Conference on Biomedical Engineering and Sciences (IECBES), 2012, pp. 636-641.

[51] S. Lemm, B. Blankertz, G. Curio, and K. Muller, "Spatio-spectral filters for improving the classification of single trial EEG," IEEE Transactions on Biomedical Engineering, vol. 52, pp. 1541-1548, 2005.

[52]X. An, D. Kuang, X. Guo, Y. Zhao, and L. He, "A Deep Learning Method for Classification of EEG Data Based on Motor Imagery," in Intelligent Computing in Bioinformatics: 10th International Conference, ICIC 2014, Taiyuan, China, August 3-6, 2014. Proceedings, D.-S. Huang, K. Han, and M. Gromiha, Eds., ed Cham: Springer International Publishing, 2014, pp. 203-210.

[53] N. Lu, T. Li, X. Ren, and H. Miao, "A Deep Learning Scheme for Motor Imagery Classification based on Restricted Boltzmann Machines," IEEE Transactions on Neural Systems and Rehabilitation Engineering, vol. PP, pp. 1-1, 2016.

[54] G. Dornhege, B. Blankertz, G. Curio, and K. Muller, "Boosting bit rates in noninvasive EEG single-trial classifications by feature combination and multiclass paradigms," IEEE Transactions on Biomedical Engineering, vol. 51, pp. 993-1002, 2004.

[55] B. Blankertz, K. R. Müller, D. J. Krusienski, G. Schalk, J. R. Wolpaw, A. Schlögl, et al., "The BCI competition III: validating alternative approaches to actual BCI problems," IEEE Transactions on Neural Systems and Rehabilitation Engineering, vol. 14, pp. 153-159, June 2006.

[56] S. Kumar, R. Sharma, A. Sharma, and T. Tsunoda, "Decimation Filter with Common Spatial Pattern and Fishers Discriminant Analysis for Motor Imagery Classification " presented at the IEEE World Congress on Computational Intelligence, Vancouver, Canada, 2016. 